\title{
Local Stability and Hydrodynamical Limit of Spitzer's One Dimensional Lattice Model
}

\author{
J. Fritz \\ Mathematical Institute, H-1053 Budapest, Reáltanoda u. 13, Hungary
}

\begin{abstract}
An infinite system of ordinary differential equations is considered, the right hand side is just the negative gradient of potential energy of a onedimensional system of unbounded spins interacting by a symmetric and convex pair potential. Constant configurations are stationary points and the mean spin is conserved. It is shown that each of these stationary points has its own domain of attraction, the initial distribution need not be translation invariant. As a consequence we obtain that the mean spin satisfies the heat equation in the hydrodynamical limit.
\end{abstract}

\section{Introduction}

One of the most striking difficulties in the study of temporal evolution of large physical systems is certainly the existence of whole families of stationary states. Although degeneracy of the stationary state is usually associated to conservation principles, relaxation to equilibrium is as yet poorly understood. Perhaps the simplest but not exactly solvable model of this kind is the following gradient dynamics of one-dimensional systems of unbounded spins. Let $\mathbb{R}$ denote the real line, let $\mathbb{Z}$ be the set of integers, and suppose that we are given a continuously differentiable, symmetric and convex function $U: \mathbb{R} \rightarrow[0,+\infty)$. Elements of the product space $\mathbb{R}^{\mathbb{Z}}$ are represented as doubly infinite sequences $\omega=\left(\omega_{k}\right)_{k \notin \mathbb{Z}}$, i.e. $\omega_{k}$ denotes the $k^{\text {th }}$ co-ordinate of $\omega \in \mathbb{R}^{\mathbb{Z}}$. The purpose of this paper is to investigate asymptotic behaviour of solutions to the Cauchy problem for

$$
\frac{d \omega_{k}(t)}{d t}=-U^{\prime}\left(\omega_{k}(t)-\omega_{k-1}(t)\right)-U^{\prime}\left(\omega_{k}(t)-\omega_{k+1}(t)\right)
$$

where $t \geqq 0, k \in \mathbb{Z}$ and $U^{\prime}$ denotes the derivative of $U$. Of course, (0.1) will be considered only in an appropriately chosen subset of $\mathbb{R}^{\mathbb{Z}}$. Let us remark that symmetry of $U$ implies a conservation law for the mean spin, and configurations of type $\omega_{k}=\mu+\lambda k$ are stationary points of $(0.1)$. 
The study of first order systems like (0.1) has been initiated by Spitzer [1]. Assuming that the initial configuration is random with a translation invariant distribution, convergence to equilibrium was proven by Garcia and Kesten [2] for lattice models, and by Lang [4] for point systems. Fairly general models of this kind were investigated by Fichtner and Freudenberg in [5] and in a series of related papers. In all proofs, however, it is very important that the initial configuration is random, and its distribution is translation invariant; we are going to remove these restrictions. Unfortunately, we are able to prove local stability and the related hydrodynamical limit only for the constant configurations.

\section{Main Result}

Besides differentiability and symmetry of $U$, we are assuming that

$$
0<\frac{1}{L} \leqq \frac{U^{\prime}(x)-U^{\prime}(y)}{x-y} \leqq L<+\infty \quad \text { if } \quad x \neq y,
$$

and

$$
\left|U^{\prime}(x)-\frac{x}{2}\right| \leqq L|x|^{3}
$$

hold with some constant $L$. Notice that if $U$ is four times differentiable at $x=0$ and $U^{\prime \prime}(0)=\frac{1}{2}$, then (1.2) follows by the symmetry property $U(x)=U(-x)$ of $U$.

The infinite product space $\mathbb{R}^{\mathbb{Z}}$ is certainly too large to be the phase space for (0.1). It will be convenient to assume that the initial configuration belongs to $\Omega=\left[\omega \in \mathbb{R}^{\mathbb{Z}} ;|\omega|<\infty\right]$, where $|\cdot|$ is a norm defined by

$$
|\omega|^{2}=\sup _{m \in \mathbb{Z}} \sup _{n \geqq \sqrt{|m|+1}} \frac{1}{2 n+1} \sum_{k=m-n}^{m+n} \omega_{k}^{2} .
$$

It is easy to check that $\Omega$ is a Banach space with this norm, and $|\cdot|$ is measurable with respect to the product $\sigma$-algebra, $\mathscr{R}^{\mathbb{Z}}$, of $\mathbb{R}^{\mathbb{Z}}$, thus $\Omega \in \mathscr{R}^{\mathbb{Z}}$. We are going to consider probability measures only on $\mathscr{A}=\Omega \cap \mathscr{R}^{\mathbb{Z}}$, the Borel $\sigma$-algebra of $\Omega$ is too large because $\Omega$ is not a separable Banach space. Let us remark that $\Omega$ is of full measure with respect to a wide class of probability measures on $\left(\mathbb{R}^{\mathbb{Z}}, \mathscr{R}^{\mathbb{Z}}\right)$.

Let $F(\omega)=\left(F_{k}(\omega)\right)_{k \in \mathbb{Z}}$ denote the right hand side of $(0.1)$, then we have $|F(\omega)|$ $\leqq M|\omega|$ and $|F(\omega)-F(\bar{\omega})| \leqq M|\omega-\bar{\omega}|$ in view of (1.1) and of $U^{\prime}(0)=0$. Therefore, for each $\sigma \in \Omega$ there exists a unique solution $\omega(t, \sigma)$ of $(0.1)$ such that $\omega(0, \sigma)=\sigma$ and $|\omega(t, \sigma)| \leqq|\sigma| e^{M t}$, see e.g. [11]. The construction implies also that each coordinate, $\omega_{k}(t, \sigma)$, of the general solution is a measurable function of $\sigma$, consequently (0.1) induces a flow in the space of probability measures on $(\Omega, \mathscr{A})$, too.

Inequality (1.2) suggests that $(0.1)$ should be considered as a perturbation of the linear system

$$
\frac{d w_{k}(t)}{d t}=-w_{k}(t)+\frac{1}{2}\left[w_{k-1}(t)+w_{k+1}(t)\right], \quad k \in \mathbb{Z}
$$


Let $w(t, \sigma)$ denote the solution of (1.4) in $\Omega$ with initial configuration $\sigma \in \Omega$, then

$$
w_{m}(t, \sigma)=\sum_{k \in \mathbb{Z}} I_{k}(t) \sigma_{m-k},
$$

where

$$
I_{k}(t)=\frac{e^{-t}}{\pi} \int_{0}^{\pi} \exp (t \cos x) \cos k x d x, \quad k \in Z .
$$

Since $I_{k}(t)=w_{k}(t, \sigma)$ if $\sigma_{0}=1, \sigma_{i}=0$ otherwise, we see that

$$
I_{k}(t)=P\left[X_{t}=k \mid X_{0}=0\right],
$$

where $X_{t}$ is the symmetric random walk on $\mathbb{Z}$ with continuous time. Further, if $b(x)=U^{\prime}(x)-\frac{x}{2}$ and

$$
c_{k}(\omega)=-b\left(\omega_{k}-\omega_{k-1}\right)-b\left(\omega_{k}-\omega_{k+1}\right),
$$

then iterating the linear part of $(0.1)$ we obtain

$$
\omega_{m}(t, \sigma)=w_{m}(t, \sigma)+\int_{0}^{t} \sum_{k \in \mathbb{Z}} I_{k}(t-s) c_{m-k}(\omega(s, \sigma)) d s .
$$

In view of uniqueness of solutions, (1.5) and (1.8) can be verified by a direct calculation. Our principal result is the following inequality.

Proposition 1.9. There exists a universal constant $K$ such that

$$
\left[\omega_{m+1}(t, \sigma)-\omega_{m}(t, \sigma)\right]^{2} \leqq \frac{K}{1+t} \sum_{k \in \mathbb{Z}} \sigma_{k}^{2} \exp \left[-\frac{|k-m|}{K \sqrt{1+t}}\right] .
$$

Comparing (1.2), (1.8), and (1.9) we obtain that (0.1) is an asymptotically negligible perturbation of (1.4), at least for $\sigma \in \Omega$.

Theorem 1.10. For each $m \in \mathbb{Z}$ and $t>0$ we have

$$
t^{1 / 4}\left|\omega_{m}(t, \sigma)-w_{m}(t, \sigma)\right| \leqq A|\sigma|^{3}+A|\sigma|^{2}\left[1+\frac{|m|}{\sqrt{1+t}}\right]^{1 / 2} \log (e+t)
$$

with a universal constant $A>0$.

Since the mean spin is a conserved quantity, we expect that solutions with a Cesaro-summable initial configuration converge to the corresponding constant configuration as time goes to infinity. Let $\Omega_{\mu}$ denote the set of such $\omega \in \Omega$ that $(2 n+1)^{-1} S_{0}(\omega, n) \rightarrow \mu$ as $n \rightarrow+\infty$, where

$$
S_{m}(\omega, n)=\omega_{m-n}+\omega_{m-n+1}+\ldots+\omega_{m+n} .
$$

Observe that $\left|\omega_{k}\right| \leqq 2|\omega|(1+|k|)^{1 / 4}$, thus $\omega \in \Omega_{\mu}$ implies

$$
\lim (2 n+1)^{-1} S_{m}(\omega, n)=\mu
$$

for each $m$. Each $\Omega_{\mu}$ is an invariant set of our dynamics, i.e. $\omega(t, \sigma) \in \Omega_{\mu}$ for all $t>0$ if $\sigma \in \Omega_{\mu}$. Asymptotics of $w_{m}(t, \sigma)$ is easily calculable, thus (1.10) yields 
Theorem 1.11. Let $\sigma \in \Omega_{\mu}$, then for each $m \in \mathbb{Z}$ we have $\omega_{m}(t, \sigma) \rightarrow \mu$ as $t \rightarrow+\infty$. Moreover, if

$$
\limsup _{n \rightarrow+\infty}[\sqrt{n} \log n]^{-1}\left|S_{m}(\sigma, n)-(2 n+1) \mu\right|<+\infty
$$

then

$$
\limsup _{t \rightarrow+\infty} \frac{t^{1 / 4}}{\log t}\left|\omega_{m}(t, \sigma)-\mu\right|<+\infty .
$$

If the initial configuration is not Cesaro-summable, then asymptotic behaviour of solutions may be very complicated. An intuitive picture can only be obtained in the hydrodynamical limit. We are going to rescale space and time according to $m \rightarrow m / h, t \rightarrow t / h^{2}$, where $h>0$ goes to zero. The scaling parameter $h$ can be interpreted as the real distance of neighbouring lattice sites. Of course, $(0.1)$ is a lattice approximation of the linear heat equation, thus the above procedure turns to be an approximation of the associated semigroup of linear operators, see [12] with some further references. Due to (1.9), however, we can prove somewhat stronger results than consequences of the general theory.

Suppose that we are given a family $P_{h}, h>0$ of probability measures on $(\Omega, \mathscr{A})$, and let

$$
\mu_{h}(t, m)=\int \omega_{m}(t, \sigma) P_{h}(d \sigma)
$$

We are assuming that for each $x \in \mathbb{R}$ we have

$$
\lim _{h \rightarrow 0} \mu_{h}(0,[x / h])=\varrho(x),
$$

where $\varrho: \mathbb{R} \rightarrow \mathbb{R}$ is a continuous function, $[u]$ denotes the integer part of $u$. Let $\varphi_{t}(x)$ denote the fundamental solution of the heat equation, i.e.

$$
\varphi_{t}(x)=(2 \pi t)^{-1 / 2} \exp \left(-\frac{x^{2}}{2 t}\right)
$$

and set $M(h)=\left(M_{m}^{2}(h)\right)_{m \in \mathbb{Z}}$, where $M_{m}^{2}(h)=\int\left|\sigma_{m}\right|^{3} P_{h}(d \sigma)$.

Theorem 1.15. Suppose that $\left|\mu_{h}(0,[x / h])\right| \leqq g(x)$ and $M(h) \in \Omega$ for each $h>0$. If $\int\left(1+x^{2}\right)^{-1} g(x) d x<+\infty$ and $\lim \sqrt{h}|M(h)|^{2}=0$ as $h \rightarrow 0$, then (1.13) implies

$$
\lim _{h \rightarrow 0} \mu_{h}\left(t / h^{2},[x / h]\right)=\int \varphi_{t}(x-y) \varrho(y) d y
$$

for each $x \in \mathbb{R}$ and $t \geqq 0$.

\section{Proof of Proposition 1.9}

A method of Liapunov functions will be used. Sums like $\sum \omega_{k}^{2}$ and $\sum U\left(\omega_{k+1}-\omega_{k}\right)$ are known to have some nice properties, see [1,4]. Since the initial distribution is not necessarily a translation invariant one, a uniform estimate of boundary effects should be given, cf. $[3,8]$. 
Let $f: \mathbb{R} \rightarrow[0,1]$ be a continuously differentiable non-increasing function such that $f(x)=1$ if $x \leqq 1, f$ is concave if $x \leqq 3, f$ is convex for $x \geqq 2$ and $f(x)=e^{2-x}$ if $x \geqq 3$. Then $f$ is linear for $2 \leqq x \leqq 3$, and $f(x) \leqq e^{2-x}$, further $0 \leqq-f^{\prime}(x) \leqq f(x)$ $\leqq e f(x+1)$, and

$$
|f(x)-f(y)| \leqq-\left(f^{\prime}(x)+f^{\prime}(y)\right)|x-y| \text { if }|x-y| \leqq 1
$$

hold, too. Our auxiliary functions, $Q$ and $H$, are defined for $\omega \in \Omega, m \in \mathbb{Z}, r \geqq 1$ by

$$
\begin{gathered}
Q_{m}(\omega, r)=\sum_{k \in \mathbb{Z}} f_{k-m}(r) \omega_{k}^{2}, \\
H_{m}(\omega, r)=\sum_{k \in \mathbb{Z}} f_{k-m}(r)\left[U\left(\omega_{k}-\omega_{k-1}\right)+U\left(\omega_{k}-\omega_{k+1}\right)\right],
\end{gathered}
$$

where

$$
f_{k}(r)=\sum_{i \in \mathbb{Z}} f\left(|k-i| r^{-1}\right) e^{-2|i|} .
$$

Notice that $0<f_{k}(r)<2, f_{k}(r)>1$ if $|k| \leqq r$, each $f_{k}$ is a non-decreasing function of $r$, and for $r \geqq 1$ we have $f_{k}(r) \leqq \exp \left(3-\frac{|k|}{r}\right)$. Only the last inequality needs a proof, we have

$$
f_{k}(r) \leqq \sum e^{-2|i|} \exp \left(2-\frac{|k-i|}{r}\right) \leqq \exp \left(-\frac{|k|}{r}\right) \sum \exp (2-|i|) \leqq e^{3} \exp \left(-\frac{|k|}{r}\right)
$$

as $r \geqq 1$ and $-|k-i| \leqq-|k|+|i|$. The proof of (1.9) is based on the following property of $f_{k}(r)$.

$$
\left[f_{k+1}(r)-f_{k}(r)\right]^{2} \leqq 2 \frac{e^{2}}{r}\left[f_{k}(r)+f_{k+1}(r)\right] \min \left[f_{k}^{\prime}(r), f_{k+1}^{\prime}(r)\right] .
$$

Indeed, from (2.1) we obtain that

$$
\begin{aligned}
\left|f_{k+1}(r)-f_{k}(r)\right| & \leqq \sum_{i \in \mathbb{Z}} e^{-2|i|}\left|f\left(\frac{|k+1-i|}{r}\right)-f\left(\frac{|k-i|}{r}\right)\right| \\
& \leqq-\frac{1}{r} \sum_{i \in \mathbb{Z}} e^{-2|i|}\left[f^{\prime}\left(\frac{|k-i|}{r}\right)+f^{\prime}\left(\frac{|k+1-i|}{r}\right)\right],
\end{aligned}
$$

thus by $-f^{\prime} \leqq f$ we get

$$
\begin{aligned}
\left|f_{k+1}(r)-f_{k}(r)\right| & \leqq \\
r & \sum_{i \in \mathbb{Z}} e^{-2|i|}\left[f\left(\frac{|k-i|}{r}\right)+f\left(\frac{\mid k+1-i}{r}\right)\right] \\
& =\frac{1}{r}\left[f_{k}(r)+f_{k+1}(r)\right] .
\end{aligned}
$$

On the other hand,

$$
f_{k}^{\prime}(r)=-\sum_{i \in \mathbb{Z}} e^{-2|i|} f^{\prime}\left(\frac{|k-i|}{r}\right)|k-i| r^{-2} \geqq-\frac{1}{r} \sum_{i \in \mathbb{Z}} e^{-2|i|} f^{\prime}\left(\frac{|k-i|}{r}\right)
$$


as $|k-i| \geqq r$ if $f^{\prime}\left(|k-i| r^{-1}\right) \neq 0$, and

$$
\begin{aligned}
f_{k}^{\prime}(r) & =-\sum_{i \in \mathbb{Z}} e^{-2|k-i|} f^{\prime}\left(\frac{|i|}{r}\right)|i| r^{-2} \\
& \leqq e^{2|j-k|} \sum_{i \in \mathbb{Z}} e^{-2|j-i|} f^{\prime}\left(\frac{|i|}{r}\right)|i| r^{-2}=e^{2|j-k|} f_{j}^{\prime}(r) .
\end{aligned}
$$

Applying (2.8) and (2.9) to the product of (2.6) and (2.7) we obtain (2.5).

Suppose now that $\omega=\omega(t)$ is a solution of $(0.1)$, then

$$
\begin{aligned}
\frac{\partial}{\partial t} Q_{m}(\omega(t), r)= & -2 \sum_{k \in \mathbb{Z}} f_{k-m}(r) \omega_{k}\left[U^{\prime}\left(\omega_{k}-\omega_{k-1}\right)+U^{\prime}\left(\omega_{k}-\omega_{k+1}\right]\right. \\
= & -2 \sum_{k \in \mathbb{Z}}\left[f_{k+1-m}(r) \omega_{k+1}-f_{k-m}(r) \omega_{k}\right] U^{\prime}\left(\omega_{k+1}-\omega_{k}\right) \\
= & -\sum_{k \in \mathbb{Z}}\left[f_{k+1-m}(r)-f_{k-m}(r)\right]\left(\omega_{k}+\omega_{k+1}\right) U^{\prime}\left(\omega_{k+1}-\omega_{k}\right) \\
& -\sum_{k \in \mathbb{Z}}\left[f_{k+1-m}(r)+f_{k-m}(r)\right]\left(\omega_{k+1}-\omega_{k}\right) U^{\prime}\left(\omega_{k+1}-\omega_{k}\right)
\end{aligned}
$$

Since $\left|U^{\prime}(x)\right| \leqq L|x|$ and $x U^{\prime}(x) \geqq \frac{1}{L} x^{2}$ follow from (1.1), in view of $a u-\frac{b}{2} u^{2} \leqq a^{2} / 2 b$ we have

$$
\begin{aligned}
& -\left[f_{k+1-m}(r)-f_{k-m}(r)\right]\left(\omega_{k+1}+\omega_{k}\right) U^{\prime}\left(\omega_{k+1}-\omega_{k}\right) \\
& -\frac{1}{2}\left[f_{k+1-m}(r)+f_{k-m}(r)\right]\left(\omega_{k+1}-\omega_{k}\right) U^{\prime}\left(\omega_{k+1}-\omega_{k}\right) \\
& \leqq \frac{L^{3}}{2}\left[f_{k+1-m}(r)-f_{k-m}(r)\right]^{2}\left[f_{k+1-m}(r)+f_{k-m}(r)\right]^{-1}\left(\omega_{k+1}+\omega_{k}\right)^{2} .
\end{aligned}
$$

Therefore $(2.5)$ and $(u+v)^{2} \leqq 2 u^{2}+2 v^{2}$ imply

$$
\frac{\partial}{\partial t} Q_{m}(\omega(t), r)+\sum_{k \in \mathbb{Z}} f_{k-m}(r)\left(\omega_{k+1}-\omega_{k}\right) U^{\prime}\left(\omega_{k+1}-\omega_{k}\right) \leqq 4 e^{2} L^{3} \frac{1}{r} \frac{\partial}{\partial r} Q_{m}(\omega(t), r)
$$

by a direct calculation.

A very similar argument applies to $H$. We may (and do) assume that $U(0)=0$. Then from (1.1) we obtain that $U^{\prime 2}(x) \leqq 2 L^{3} U(x)$. Let

$$
F_{k}(\omega)=-U^{\prime}\left(\omega_{k}-\omega_{k-1}\right)-U^{\prime}\left(\omega_{k}-\omega_{k+1}\right)
$$

denote the right hand side of $(0.1)$, and let $Z_{k}=[j \in \mathbb{Z} ;|j-k|=1]$. Exploiting symmetry of $U$ we obtain

$$
\begin{aligned}
\frac{\partial}{\partial t} H_{m}(\omega(t), r)= & \sum_{k \in \mathbb{Z}} f_{k-m}(r) \sum_{j \in Z_{k}} U^{\prime}\left(\omega_{k}-\omega_{j}\right)\left[F_{k}(\omega)-F_{j}(\omega)\right] \\
= & -\sum_{k \in \mathbb{Z}} f_{k-m}(r)\left[F_{k}(\omega)\right]^{2}-\sum_{k \in \mathbb{Z}} \sum_{j \in Z_{k}} f_{j-m}(r) U^{\prime}\left(\omega_{j}-\omega_{k}\right) F_{k}(\omega) \\
= & \sum_{k \in \mathbb{Z}} F_{k}(\omega) \sum_{j \in Z_{k}}\left[f_{k-m}(r)-f_{j-m}(r)\right] U^{\prime}\left(\omega_{j}-\omega_{k}\right) \\
& -2 \sum_{k \in \mathbb{Z}} f_{k-m}(r)\left[F_{k}(\omega)\right]^{2}
\end{aligned}
$$




$$
\begin{aligned}
& \leqq \frac{1}{8} \sum_{k \in \mathbb{Z}}\left[f_{k-m}(r)\right]^{-1}\left[\sum_{j \in Z_{k}}\left(f_{k-m}(r)-f_{j-m}(r)\right) U^{\prime}\left(\omega_{j}-\omega_{k}\right)\right]^{2} \\
& \leqq 2 L^{3} \sum_{k \in \mathbb{Z}} \sum_{j \in Z_{k}} \frac{\left[f_{k-m}(r)-f_{j-m}(r)\right]^{2}}{f_{k-m}(r)+f_{j-m}(r)} U\left(\omega_{k}-\omega_{j}\right)
\end{aligned}
$$

Thus from (2.5) we have

$$
\frac{\partial}{\partial t} H_{m}(\omega(t), r) \leqq 4 e^{2} L^{3} \frac{1}{r} \frac{\partial}{\partial r} H_{m}(\omega(t), r) .
$$

Therefore, if $r=r(t)=\left[1+8 e^{2} L^{3}(T-t)\right]^{1 / 2}$ for $t \leqq T$, then $H_{m}(\omega(t), r(t))$ and $Q_{m}(\omega(t), r(t))$ are decreasing functions of $t \leqq T$, and (2.12) turns into

$$
\begin{aligned}
& \int_{0}^{T} \sum_{k \in \mathbb{Z}} f_{k-m}(r(t))\left[\omega_{k+1}(t)-\omega_{k}(t)\right] U^{\prime}\left(\omega_{k+1}(t)-\omega_{k}(t)\right) d t \\
& \quad \leqq Q_{m}\left(\omega(0),\left(1+8 e^{2} L^{3} T\right)^{1 / 2}\right) .
\end{aligned}
$$

On the other hand, $x U^{\prime}(x) \geqq 2 L^{-2} U(x)$, and $H_{m}(\omega(t), r(t))$ decreases. Consequently

$$
T H_{m}(\omega(T), 1) \leqq e L^{2} Q_{m}\left(\omega(0),\left(1+8 e^{2} L^{3} T\right)^{1 / 2}\right) .
$$

Since $U(x) \geqq x^{2} / 2 L$ and $f_{k}(r) \leqq \exp \left(3-\frac{|k|}{r}\right),(2.16)$ implies (1.9), at least if $t>1$. For small values of $t$, however, (1.9) follows directly from the monotonocity of $Q_{m}(\omega(t), r(t))$.

\section{Proof of Theorem 1.10}

Observe first that (1.9) implies

$$
\left[\omega_{m+1}(t, \sigma)-\omega_{m}(t, \sigma)\right]^{2} \leqq K_{1} \frac{|\sigma|^{2}}{1+t} \max [\sqrt{1+t}, \sqrt{1+|m|}]
$$

by an easy calculation that is the same as the proof of (2.17) in [8]. We are going to estimate the integral on the right hand side of (1.8) by means of (3.1).

Since $c(\omega)=\left(c_{k}(\omega)\right)_{k \in \mathbb{Z}}$, see $(1.7)$, is an element of $\Omega,(1.8)$ can be rearranged as follows

$$
\omega_{m}(t, \sigma)-w_{m}(t, \sigma)=\int_{0}^{t} \sum_{n=0}^{\infty}\left[I_{n}(t-s)-I_{n+1}(t-s)\right] S_{m}(c(\omega(s, \sigma)), n) d s,
$$

where, just as earlier, $S_{m}(c, n)=c_{m-n}+c_{m-n+1}+\ldots+c_{m+n}$. To evaluate (3.2) some simple properties of the $I_{n}(t)$ are needed. Expanding the exponential function into its power series we obtain

$$
I_{n}(t)=e^{-t} \sum_{m=0}^{\infty} \frac{\left(\frac{t}{2}\right)^{2 m+n}}{m !(n+m) !}
$$


for $n \geqq 0$, and $I_{-n}(t)=I_{n}(t)$, see 8.445 in [10]. Hence it follows easily that $I_{n}(t) \geqq 0$ and

$$
\sum_{n \in \mathbb{Z}} I_{n}(t)=1
$$

the series in (3.4) converges faster than exponentially. It will be very useful that

$$
I_{n+1}(t) \leqq I_{n}(t), \quad \text { if } \quad n \geqq 0 .
$$

Though I could not find (3.5) in textbooks on Bessel functions, the following proof is due to Elbert [9]. The inequality for arithmetic and geometric means implies that

$$
\frac{\left(\frac{t}{2}\right)^{2 m+n+1}}{m !(n+m+1) !} \leqq \frac{1}{2} \frac{\left(\frac{\mathrm{t}}{2}\right)^{2 m+n}}{\mathrm{~m} !(n+m) !}+\frac{1}{2} \frac{\left(\frac{t}{2}\right)^{2 m+n+2}}{(m+1) !(n+m+1) !}
$$

whence, by summing over $m$ we obtain (3.5). Aymptotics of $I_{n}(t)$ is also interesting; since $\cos x-1 \leqq-x^{2} / 16$ if $0 \leqq x \leqq \pi$, from (1.6) we obtain that $I_{0}(t) \leqq 2 / \sqrt{t}$ for $t>0$, and the best lower bound is $(2 \pi t)^{-1 / 2}$.

Now we are in a position to conclude (1.10). Since $b(x)=-b(-x)$, we have

$$
S_{m}(c(\omega), n)=b\left(\omega_{m+n+1}-\omega_{m+n}\right)-b\left(\omega_{m-n}-\omega_{m-n-1}\right),
$$

but $|b(x)| \leqq(L+1) x^{2}$ in view of (1.1) and (1.2). Thus (3.1) results in

$$
\left|S_{m}(c(\omega(s, \sigma)), n)\right| \leqq K_{2}|\sigma|^{3}(1+s)^{-3 / 4}
$$

if $s \geqq|m|+n$, cf. (1.2), while

$$
\left|S_{m}(c(\omega(s, \sigma)), n)\right| \leqq K_{3}|\sigma|^{2} \frac{\sqrt{1+|m|+n}}{1+s}
$$

otherwise; $K_{2}$ and $K_{3}$ depend only on $L$. Since $I_{n}(t-s) \geqq I_{n+1}(t-s)$ if $n \geqq 0$ and $0 \leqq s \leqq t, I_{0}(t-s) \leqq 2(t-s)^{-1 / 2}$ if $0 \leqq s \leqq t$, and

$$
\int_{0}^{t}(t-s)^{-1 / 2}(1+s)^{-3 / 4} d s \leqq 9(1+t)^{-1 / 4}
$$

if $t \geqq 0$; the case (3.7) yields a contribution not larger than $18 K_{2}|\sigma|^{3}(1+t)^{-1 / 4}$ to (3.2). Therefore, it suffices to estimate

$$
J=\int_{0}^{t} \sum_{n=0}^{\infty}\left[I_{n}(t-s)-I_{n+1}(t-s)\right] \frac{\sqrt{1+|m|+n}}{1+s} d s .
$$

Let $u=[\sqrt{t}]$, then

$$
\begin{aligned}
J \leqq & \int_{0}^{t} \sum_{n=0}^{u}\left[I_{n}(t-s)-I_{n+1}(t-s)\right] \frac{\sqrt{1+|m|+u}}{1+s} d s \\
& +\int_{0}^{t} \sum_{n=u+1}^{\infty}\left[I_{n}(t-s)-I_{n+1}(t-s)\right] \frac{\sqrt{1+|m|+n}}{1+s} d s \\
\leqq & \sqrt{1+|m|+u} \int_{0}^{t}\left[I_{0}(t-s)-I_{u+1}(t-s)\right] \frac{1}{1+s} d s
\end{aligned}
$$




$$
\begin{aligned}
& +\int_{0}^{t} \sum_{n=u+1}^{\infty}\left[I_{n}(t-s)-I_{n+1}(t-s)\right] \frac{\sqrt{1+|m|+n}}{1+s} d s \\
\leqq & \sqrt{1+|m|+u} \int_{0}^{t} I_{0}(t-s) \frac{1}{1+s} d s+\frac{1}{2} \int_{0}^{t} \sum_{n=u}^{\infty} I_{n+1}(t-s) \frac{1}{(1+s) \sqrt{1+|m|+n}} d s,
\end{aligned}
$$

as $\sqrt{1+x}-\sqrt{x} \leqq 1 / 2 \sqrt{x}$ if $x>0$. But

$$
\int_{0}^{t} \frac{d s}{(1+s) \sqrt{t-s}} \leqq \frac{\sqrt{2}(1+\log (1+t))}{\sqrt{1+t}}
$$

and $\sum I_{n}<1$, consequently

$$
J \leqq 3(1+\log (1+t))\left[\frac{1+|m|+\sqrt{t}}{1+t}\right]^{1 / 2}+\log (1+t)(1+|m|+\sqrt{t})^{-1 / 2},
$$

which completes the proof of (1.10).

\section{Proof of Theorem 1.11}

In view of (1.10) only solutions of (1.4) should be analyzed. From (1.5) we obtain

$$
w_{m}(t, \sigma)=\sum_{n=0}^{\infty}\left[I_{n}(t)-I_{n+1}(t)\right] S_{m}(\sigma, n)
$$

Because of (3.4) we may assume that $\mu=0$. Then for each $\varepsilon>0$ there exists a positive integer $N$ such that for $n \geqq N$ we have $\left|S_{m}(\sigma, n)\right| \leqq(2 n+1) \varepsilon$, while $\left|S_{m}(\sigma, n)\right|$ $\leqq K_{N}<+\infty$ if $n<N$. Therefore

$$
\begin{aligned}
\left|w_{m}(t, \sigma)\right| & \leqq I_{0}(t) K_{N}+\varepsilon \sum_{n=0}^{\infty}\left[I_{n}(t)-I_{n+1}(t)\right](2 n+1) \\
& \leqq 2 K_{N} / \sqrt{t}+\varepsilon
\end{aligned}
$$

which proves the first statement of (1.11).

Suppose now that

$$
\left|S_{m}(\sigma, n)\right| \leqq K_{m}(1+\log (1+n)) \sqrt{1+n},
$$

and let $u=[\sqrt{t}], h(n)=(1+\log (1+n)) \sqrt{1+n} ;$ since

$$
h(n+1)-h(n) \leqq 3(1+\log (1+n))(1+n)^{-1 / 2},
$$

we obtain that

$$
\begin{aligned}
\left|w_{m}(t, \sigma)\right| & \leqq K_{m} h(u)\left[I_{0}(t)-I_{u+1}(t)\right]+K_{m} \sum_{n=u+1}^{\infty}\left[I_{n}(t)-I_{n+1}(t)\right] h(n) \\
& \leqq K_{m} h(u) I_{0}(t)+3 K_{m} \sum_{n=u}^{\infty} I_{n+1}(t)(1+\log (1+n))(1+n)^{-1 / 2} \\
& \leqq 2 K_{m} h(u) / \sqrt{t}+3 K_{m}(1+\log (1+u))(1+u)^{-1 / 2},
\end{aligned}
$$

which implies the second statement of (1.11). 


\section{Proof of Theorem $\mathbf{1 . 1 5}$}

This proof is essentially the same as that of $(1.11)$. Let $K(h)=\left(K_{m}(h)\right)_{m \in \mathbb{Z}}$, where $K_{m}^{2}(h)=\int \sigma_{m}^{2} P_{h}(d \sigma)$ and observe that $|K(h)| \leqq|M(h)|^{2 / 3}$. Indeed, by Hölder's inequality we obtain that for $n \geqq \sqrt{1+|m|}$

$$
\begin{aligned}
\sum_{k=m-n}^{m+n} K_{k}^{2}(h) & \leqq \sum_{k=m-n}^{m+n}\left[M_{k}^{2}(h)\right]^{2 / 3} \leqq(2 n+1)^{1 / 3}\left(\sum_{k=m-n}^{m+n} M_{k}^{2}(h)\right)^{2 / 3} \\
& \leqq(2 n+1)|M(h)|^{4 / 3},
\end{aligned}
$$

which proves the statement. Therefore, taking the expectation of both sides of (3.2) and following the proof of (1.10), we obtain from (1.9) for $0<h<\sqrt{t}$ that

$$
\begin{aligned}
& \int\left|\omega_{m}\left(t / h^{2}, \sigma\right)-w_{m}\left(t / h^{2}, \sigma\right)\right| P_{h}(d \sigma) \\
& \quad \leqq B t^{-1 / 4} \sqrt{h}\left(|M(h)|^{2}+|M(h)|^{4 / 3}\left[1+\frac{|m h|}{\sqrt{t}}\right]^{1 / 2} \log \left(1+t / h^{2}\right)\right),
\end{aligned}
$$

where $B$ depends only on $L$.

On the other hand, let $v_{h}(t, m)=\int w_{m}(t, \sigma) P_{h}(d \sigma)$, and notice that $v_{h}$ satisfies (1.4) with initial condition $v_{h}(0, m)=\mu_{h}(0, m)$. Therefore

$$
v_{h}\left(t / h^{2},[x / h]\right)=\int_{-\infty}^{+\infty} G_{h}\left(t / h^{2},\left[\frac{x-y}{h}\right]\right) \mu(0,[y / h]) d y,
$$

where $G_{h}(s, n)=\frac{1}{h} I_{n}(s)$. The local version of the central limit theorem, see [13], implies that

$$
\lim _{h \rightarrow 0} G_{h}\left(t / h^{2},\left[\frac{x-y}{h}\right]\right)=\varphi_{t}(x-y) .
$$

Thus

$$
G_{h}\left(t / h^{2},\left[\frac{x-y}{h}\right]\right) \leqq \frac{4 \sqrt{t}}{(x-y)^{2}}
$$

means that the convergence of the integrand of $(5.3)$ to $\varphi_{t}(x-y) \varrho(y)$ is a dominated one; consequently

$$
\lim _{h \rightarrow 0} v_{h}\left(t / h^{2},[x / h]\right)=\int_{-\infty}^{+\infty} \varphi_{t}(x-y) \varrho(y) d y .
$$

Comparing (5.2) and (5.6) we obtain (1.11). To show (5.5) let us consider (1.6). If $n>0$, then integrating by parts we obtain that

$$
\begin{aligned}
I_{n}(t) & =\frac{t e^{-t}}{n \pi} \int_{0}^{\pi} \sin x e^{t \cos x} \sin n x d x \\
& =\frac{t e^{-t}}{n^{2} \pi} \int_{0}^{\pi} \cos x e^{t \cos x} \cos n x d x-\frac{t^{2} e^{-t}}{n^{2} \pi} \int_{0}^{\pi} \sin ^{2} x e^{t \cos x} \cos n x d x \\
& \leqq-\frac{t e^{-t}}{n^{2} \pi} \int_{0}^{\pi} \sin x\left(e^{t \cos x}\right)^{\prime} d x+\frac{t}{n^{2}} I_{0}(t) \leqq \frac{2 t}{n^{2}} I_{0}(t) \leqq \frac{4 \sqrt{t}}{n^{2}},
\end{aligned}
$$

which proves (5.5) and completes the proof of (1.11). 
Remark 5.8. A nontrivial hydrodynamical limit is expected if the initial configuration is close to $\omega_{k}=\mu+\lambda k$ with $\lambda \neq 0$. The method of (1.9) yields

$$
\limsup _{t \rightarrow+\infty} \sqrt{t}\left[2 \omega_{m}(t, \sigma)-\omega_{m-1}(t, \sigma)-\omega_{m+1}(t, \sigma)\right]^{2} \leqq K|\sigma|^{2}
$$

in such situations. This problem will be discussed in a forthcoming paper.

\section{References}

1. Spitzer, F.: Random processes defined through the interaction of an infinite particle system. In: Lecture Notes in Mathematics, Vol. 89, 201-223. Berlin, Heidelberg, New York: Springer 1969

2. Garcia, A., Kesten, H.: Unpublished, see footnote on p. 221 in [1]

3. Dobrushin, R.L., Fritz, J.: Non-equilibrium dynamics of one-dimensional infinite particle systems with a hard-core interaction. Commun. Math. Phys. 55, 275-292 (1977)

4. Lang, R.: On the asymptotic behaviour of infinite gradient systems. Commun. Math. Phys. 65, 129-149 (1979)

5. Fichtner, K.H., Freudenberg, W.: Asymptotic behaviour of time evolutions of infinite particle systems. Z. Wahrsch. Verw. Gebiete 54, 141-159 (1980)

6. Boldrighini, C., Dobrushin, R.L., Souhov, Ju.M.: The asymptotics of some degenerate models for the evolution of infinite particle systems. Itogi Nauki Tekh. 14, 148-255 Moscow VINITI (1979) (in Russian)

7. Boldrighini, C., Dobrushin, R.L., Souhov, Ju.M.: Hydrodynamical limit for a degenerate model of classical statistical mechanics. (in Russian) (to appear)

8. Fritz, J.: Infinite lattice systems of interacting diffusion processes, Existence and regularity properties. Z. Wahrsch. Verw. Gebiete 59, 291-309 (1980)

9. Elbert, A.: Private communication

10. Gradshtein, I.S., Ryzhik, I.M.: Table of integrals, series and products. New York, London, Toronto, Sydney, San Francisco: Academic Press 1980

11. Daleckii, J.L., Krein, M.G.: Stability of solutions to differential equations in Banach spaces. Moscow: Nauka 1970 (in Russian)

12. Kato, T.: Perturbation theory for linear operators. Berlin, Heidelberg, New York: Springer 1980

13. Feller, W.: An introduction to probability theory and its applications. Vol. II. New York, London, Sydney: Wiley 1966

Communicated by J. L. Lebowitz

Received April 5, 1982 
